\title{
Ar ion induced copper germanide phase formation at room temperature
}

\author{
S DHAR, T SOM, Y N MOHAPATRA and V N KULKARNI*
}

Department of Physics, Indian Institute of Technology, Kanpur 208016 , India

\begin{abstract}
The copper germanide phase $\mathrm{Cu}_{3} \mathrm{Ge}$ which is emerging as an alternative material for making contacts and interconnects for semiconductor industry has been produced across the interface of $\mathrm{Cu} / \mathrm{Ge}$ bilayers by ion beam mixing at room temperature using $1 \mathrm{MeV}$ Ar ions. The dose dependence of the thickness of the mixed region shows a diffusion controlled mixing process. The experimental mixing rate and efficiency for this phase are $5.35 \mathrm{~nm}^{4}$ and $10.85 \mathrm{~nm}^{5} / \mathrm{keV}$ respectively. At doses above $8 \times 10^{15} \mathrm{Ar} / \mathrm{cm}^{2}$ the formation and growth of another copper rich phase $\mathrm{Cu}_{5} \mathrm{Ge}$ has been observed. The present theoretical models are inadequate to explain the observed experimental mixing rate.
\end{abstract}

Keywords. Ions; interconnections.

\section{Introduction}

In the past two decades, there has been intensive search for suitable contact and interconnect materials for packaging very large number of semiconductor devices on smaller area (Nastasi and Mayer 1994). In this connection, it has been reported that the copper germanide $\left(\mathrm{Cu}_{3} \mathrm{Ge}\right)$ phase, produced by thermal annealing, possesses most of the suitable properties (low resistivity, low diffusivity etc.) required for contacts or interconnects in the existing technology (Krusin-Elbaum and Aboelfotoh 1991; Aboelfotoh et al 1994). We have recently shown that this copper germanide phase can be synthesized at room temperature by ion beam mixing of multilayer configuration of $\mathrm{Cu}$ and $\mathrm{Ge}$ using $\mathrm{MeV} \mathrm{Kr}$ ion beams (Dhar et al 1995). We have also shown that $\mathrm{Cu}_{3} \mathrm{Ge}$ phase forms prior to the formation of $\mathrm{Cu}_{5} \mathrm{Ge}$ phase and that kinetics play a dominant role in determining the sequence of phase formation. In this paper we explore the possibility of formation of copper germanide phases by $\mathrm{MeV}$ Ar ion induced mixing of $\mathrm{Cu}$ and $\mathrm{Ge}$ in bilayer configuration. The dose dependence of the growth of the $\mathrm{Cu}_{3} \mathrm{Ge}$ phase has been studied. The mixing rate has been compared with those predicted by existing models (Sigmund and Gras-Marti 1981; Cheng 1990; Desimoni and Traverse 1993).

\section{Experimental}

Prior to deposition, all high purity quartz substrates were cleaned properly to remove the surface contaminants. The high purity $\mathrm{Cu}$ and $\mathrm{Ge}$ films were sequentially deposited at the rate of $5 \AA / s$ on these clean substrates using an e-beam evaporation unit to produce the bilayer structure for ion beam mixing experiments. A calibrated thickness monitor was used for measuring thickness of the films during deposition.

Ion beam mixing of the samples were carried out at room temperature by $1 \mathrm{MeV} \mathrm{Ar}^{+}$ ions obtained from $2 \mathrm{MeV}$ Van-de-Graaff accelerator at the Department of Physics, IIT, Kanpur. The samples were irradiated at several doses in the range of $(2-10) \times 10^{15} \mathrm{Ar}^{+} / \mathrm{cm}^{2}$. The flux during irradiation was kept low $\left(\leqslant 1 \times 10^{12}\right.$

\footnotetext{
*Author for correspondence
} 
at. $/ \mathrm{cm}^{2} / \mathrm{sec}$ ) to ensure minimum rise in sample temperature. The amount of $\mathrm{Cu}-\mathrm{Ge}$ mixing and the composition of the mixed layers were determined by Rutherford backscattering (RBS) measurements using $1 \cdot 3 \mathrm{MeV} \mathrm{He}^{+}$ions obtained from the same accelerator. The RBS spectra before and after irradiation were analyzed using the RUMP simulation code (Doolittle 1985). The phases produced after irradiation were identified by $\mathrm{X}$-ray diffraction analysis.

\section{Results}

The RBS and X-ray analysis of the mixed bilayer samples reveal that an intermetallic phase $\mathrm{Cu}_{3} \mathrm{Ge}$ forms at the interface whose thickness increases as a function of dose up to $8 \times 10^{15} \mathrm{Ar}^{+} / \mathrm{cm}^{2}$. The RBS spectrum for a dose of $8 \times 10^{15} \mathrm{Ar} / \mathrm{cm}^{2}$ along with the spectrum of as-prepared sample is shown in figure 1.

At higher doses the copper rich phase $\mathrm{Cu}_{5} \mathrm{Ge}$ starts growing across the interface of unreacted $\mathrm{Cu}$ and $\mathrm{Cu}_{3} \mathrm{Ge}$ layer while the thickness of $\mathrm{Cu}_{3} \mathrm{Ge}$ layer reduces continuously. The comparison of the as-deposited RBS spectrum with the one irradiated at $1 \times 10^{16} \mathrm{Ar} / \mathrm{cm}^{2}$ is depicted in figure 2. This dose dependence for the growth of $\mathrm{Cu}_{3} \mathrm{Ge}$ phase is shown in figure 3 . The squared thickness $\left(X^{2}\right)$ of the mixed region of $\mathrm{Cu}_{3} \mathrm{Ge}$ phase varies linearly as a function of dose $\Phi$ up to $8 \times 10^{15} \mathrm{Ar}^{+} / \mathrm{cm}^{2}$. Note that above this dose the growth of $\mathrm{Cu}_{5} \mathrm{Ge}$ phase is concurrent with reduction in $\mathrm{Cu}_{3} \mathrm{Ge}$ phase indicating that the growth of the former phase is at the expense of $\mathrm{Cu}_{3} \mathrm{Ge}$ phase. From the slope of the curve shown in figure 3 , we get the mixing rate $\left(X^{2} / \Phi\right)$ as $5.35 \mathrm{~nm}^{4}$ for the growth of $\mathrm{Cu}_{3} \mathrm{Ge}$ phase. The mixing efficiency $\left(X^{2} / \Phi F_{\mathrm{d}}\right)$ for $\mathrm{Cu}_{3} \mathrm{Ge}$ phase is found to be $10.85 \mathrm{~nm}^{5} / \mathrm{keV}$. The value of deposited energy density $F_{\mathrm{d}}$ at the interface in this case is $50 \mathrm{eV} / \AA$ obtained from the calculations of the damage distribution using TRIM code (Ziegler et al 1986).

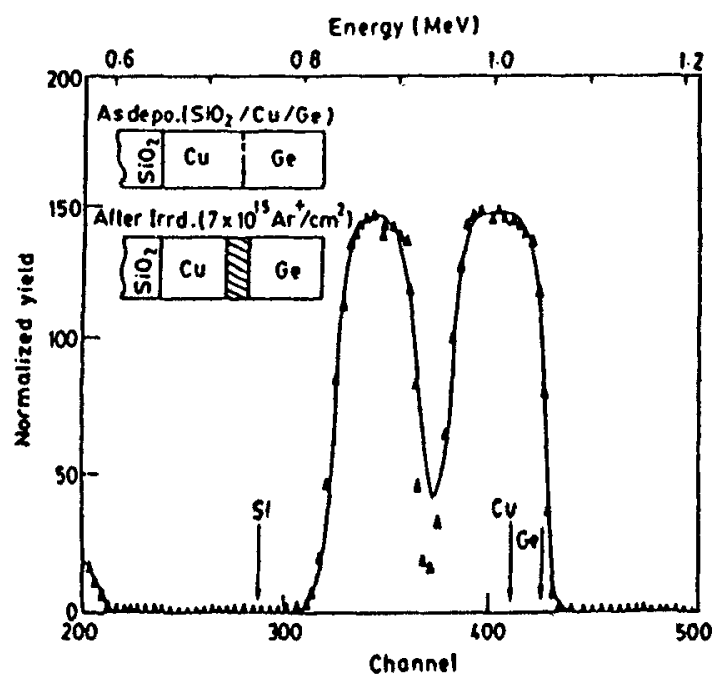

Figure 1. RBS spectra of sample $\mathrm{SiO}_{2} / \mathrm{Cu} / \mathrm{Ge}$ before $(\Delta)$ and after $(-)$ room temperature ion beam mixing at $7 \times 10^{15} \mathrm{Ar} / \mathrm{cm}^{2}$. The arrows indicate the surface position of the elements. The structures used for simulation are also shown. The hatched portions show the region of $\mathrm{Cu}_{3} \mathrm{Ge}$ phase. 


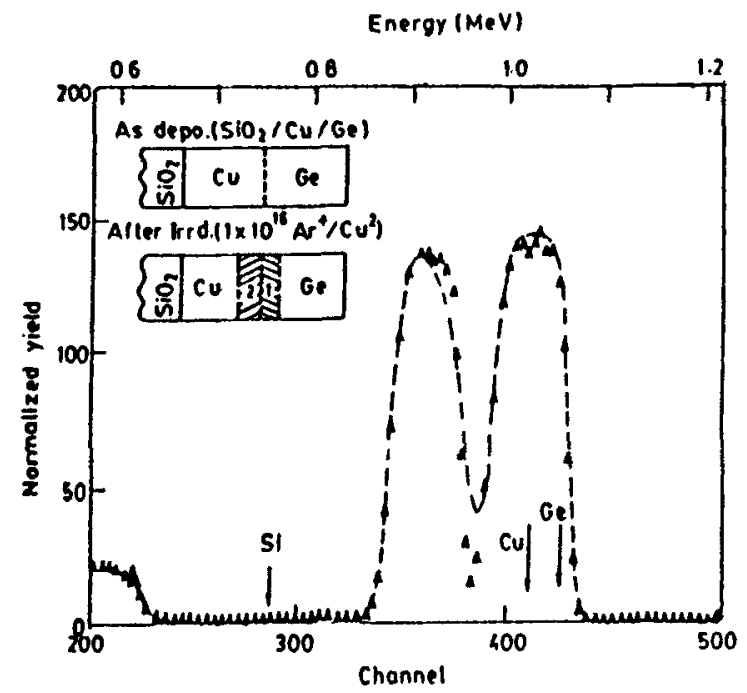

Figure 2. RBS spectra of sample $\mathrm{SiO}_{2} / \mathrm{Cu} / \mathrm{Ge}$ before ( $\Delta$ ) and after (-- ) room temperature ion beam mixing at $1 \times 10^{16} \mathrm{Ar} / \mathrm{cm}^{2}$. The arrows indicate the surface position of the elements. The structures used for simulation are also shown. The hatched portions show the region of (1) $\mathrm{Cu}_{3} \mathrm{Ge}$ phase and (2) $\mathrm{Cu}_{5} \mathrm{Ge}$ phase.

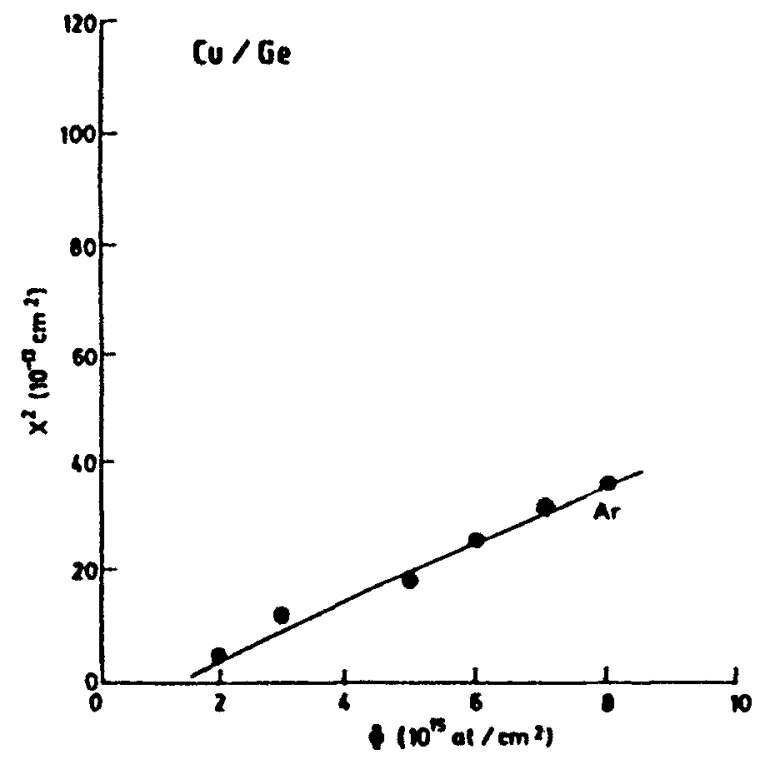

Figure 3. The squared thickness $\left(X^{2}\right)$ of the mixed layers containing $\mathrm{Cu}_{3} \mathrm{Ge}$ phase up to a dose $(\Phi)$ of $8 \times 10^{15} \mathrm{Ar} / \mathrm{cm}^{2}$. Above this dose, the growth of another $\mathrm{Cu}$ rich phase $\mathrm{Cu}_{5} \mathrm{Ge}$ occurs at the expense of the $\mathrm{Cu}_{3} \mathrm{Ge}$ phase.

\section{Discussion}

The above experimental results show that room temperature ion beam mixing of $\mathrm{Cu} / \mathrm{Ge}$ bilayers produces at first $\mathrm{Cu}_{3} \mathrm{Ge}$ phase and then $\mathrm{Cu}_{5} \mathrm{Ge}$ phase which are consistent with the results of our multilayer experiments (Dhar et al 1995) and the 
reported results of thermal annealing experiments on this system (Hong et al 1991; Dhar et al 1995). It may be noted that for most of the metal/semiconductor systems it has been found that the first phase formed by ion beam mixing is identical to the one formed in thermal annealing (Nastasi and Mayer 1994). In order to explain the mixing results of multilayer configuration of this system we have shown that $\mathrm{Cu}$ is the dominant mobile species as well as it is the limiting element, therefore, according to the effective heat of formation rule $\mathrm{Cu}_{3} \mathrm{Ge}$ phase would form first (Dhar et al 1995). The same explanation is valid for bilayer configuration as well.

The linear variation of squared thickness with dose is a signature of diffusion controlled process. This is a general result which has been obtained in many metal/metal and metal/semiconductor systems (Cheng 1990; Desimoni and Traverse 1993; Nastasi and Mayer 1994). Depending on the temperature, ion beam mixing may occur in any one of the two regions, namely, athermal region or in the thermally activated region i.e. by radiation enhanced diffusion (RED). Since, the critical temperature $T_{\mathrm{c}}$ for radiation enhanced diffusion for this system is $386 \mathrm{~K}$ (Cheng et al 1986), mixing at room temperature is occurring in the athermal region. Therefore, the contribution of RED to the mixing at room temperature irradiation would be negligible (Cheng 1990).

In the athermal region when the terminal phase is a solid solution, the mechanism of mixing can be explained either by ballistic model proposed by Sigmund and Gras-marti (1981) or Global spike model proposed by Cheng (1990). According to Ballistic model (Sigmund and Gras-marti 1981), the mixing rate depends on the ballistic properties of the incident ion and the target atoms. The ballistic mixing rate modified by Desimoni and Traverse (1993) in the case of compound formation is given by

$$
\frac{X^{2}}{\Phi_{\text {bal }}}=2\left[C_{\mathrm{Ge}} / C+4 C_{\mathrm{Cu}} / 3 C\right] \times \frac{0 \cdot 2 F_{\mathrm{d}} \lambda^{2}}{C E_{\mathrm{d}}} \times\left[\frac{4 M_{\mathrm{Cu}} M_{\mathrm{Ge}}}{\left(M_{\mathrm{Cu}}+M_{\mathrm{Ge}}\right)^{2}}\right]^{1 / 2} .
$$

Here, $M_{\mathrm{Cu}}$ and $M_{\mathrm{Ge}}$ are the atomic masses, $C$ the concentration of $\mathrm{Cu}_{3} \mathrm{Ge}$ phase, $\Delta H_{\mathrm{coh}}$ the average cohesive energy, $\lambda=10 \AA$ the minimum separation distance for stable vacancy and interstitial, $E_{\mathrm{d}}=17 \mathrm{eV}$ the average displacement energy. Using the above relationship we obtain the mixing rate of $0.85 \mathrm{~nm}^{4}$, a value which is one order of magnitude less than the experimental value. This indicates that the mixing in this case does not occur due to ballistic collisions alone but it is enhanced by strong driving force in the presence of thermal spikes. In the presence of global thermal spike the modified mixing rate (Desimoni and Traverse 1993) for compound formation can be written as

$$
\frac{X^{2}}{\Phi_{\mathrm{glo}}}=2\left[4 C_{\mathrm{Ge}} / C+4 C_{\mathrm{Cu}} / 3 C\right] \times \frac{K_{1} F_{\mathrm{d}}^{2}}{C^{5 / 3} \Delta H_{\mathrm{coh}}^{2}},
$$

where $K_{1}=0.035 \AA, C_{\mathrm{Cu}}=8.45 \times 10^{22}$ at. $/ \mathrm{cm}^{3}$ and $C_{\mathrm{Gu}}=4.42 \times 10^{22}$ at. $/ \mathrm{cm}^{3}$. Using $C=7.44 \times 10^{22}$ at. $/ \mathrm{cm}^{3}$ and $\Delta H_{\text {coh }}=3.91 \mathrm{eV}$, the global thermal spike mixing rate for compound formation calculated from the above relation is $0.34 \mathrm{~nm}^{4}$ which is again less by one order of magnitude than that of the experimental value.

This large discrepancy between experimental and calculated values shows that the mixing is not due to collisional cascades or global spike. There must be some other mechanism playing an active role during mixing in this system. Recently, Borgesen et al (1991) proposed a model for medium $Z(17 \leqslant Z \leqslant 37)$ element where solid solution 
forms due to mixing. In those cases the mixing rate can be explained by considering the formation of well separated local spikes instead of a single global spike. However, this model is not applicable in the case of present system as it considers the formation of solid solution after mixing. Nevertheless, there is a strong reason to believe that mixing is occurring in this system under local spike since it is a medium $Z$ element system where local thermal spikes are dominant. A mixing mechanism which takes into account the compound formation under local spikes would be more appropriate in this case.

\section{Conclusion}

In summary, we have shown that $\mathrm{Ar}$ ion beam mixing produces technologically important $\mathrm{Cu}_{3} \mathrm{Ge}$ phase, whose thickness varies linearly as a function of square root of the dose up to $8 \times 10^{15} \mathrm{Ar} / \mathrm{cm}^{2}$. Beyond this dose another metal rich phase $\mathrm{Cu}_{5} \mathrm{Ge}$ forms and grows in the same way at the expense of $\mathrm{Cu}_{3} \mathrm{Ge}$ phase. The mixing rate and efficiency are $5.35 \mathrm{~nm}^{4}$ and $10 \cdot 85 \mathrm{~nm}^{5} / \mathrm{keV}$ respectively for the formation of $\mathrm{Cu}_{3} \mathrm{Ge}$ phase. The existing models for ion beam mixing are unable to explain the experimentally obtained mixing rate and efficiency.

\section{References}

Aboelfotoh M O, Lin C L and Woodall J M 1994 Appl. Phys. Lett. 653245

Borgesen P, Lilienfeld D A and Masaad H 1991 Nucl. Instrum. Meth. B59/60 563

Cheng Y -T 1990 Mater. Sci. Rep. 545

Cheng Y -T, Zhao X -A, Banwell T, Workman T W, Nicolet M -A and Johnson W L 1986 J. Appl. Phys. 60 2615

Desimoni J and Traverse A 1993 Phys. Rev. B48 13266

Dhar S, Som T, Mohapatra Y N and Kulkarni V N 1995 Appl. Phys. Lett. 671700

Doolittle L R 1985 Nucl. Instrum. Meth. B9 344

Hong S Q, Comrie C M, Russel S W and Mayer J W 1991 J. Appl. Phys. 703655

Krusin -Elbaum L and Aboelfotoh M O 1991 Appl. Phys. Lett. 581341

Nastasi M and Mayer J W 1994 Mater. Sci. \& Eng. R12 1

Sigmund $P$ and Gras-marti A 1981 Nucl. Instrum. Meth. 182/183 25

Ziegler J F, Biersack J P and Littmark U 1986 The stopping and range in solids (New York: Pergamon) Vol. 1 